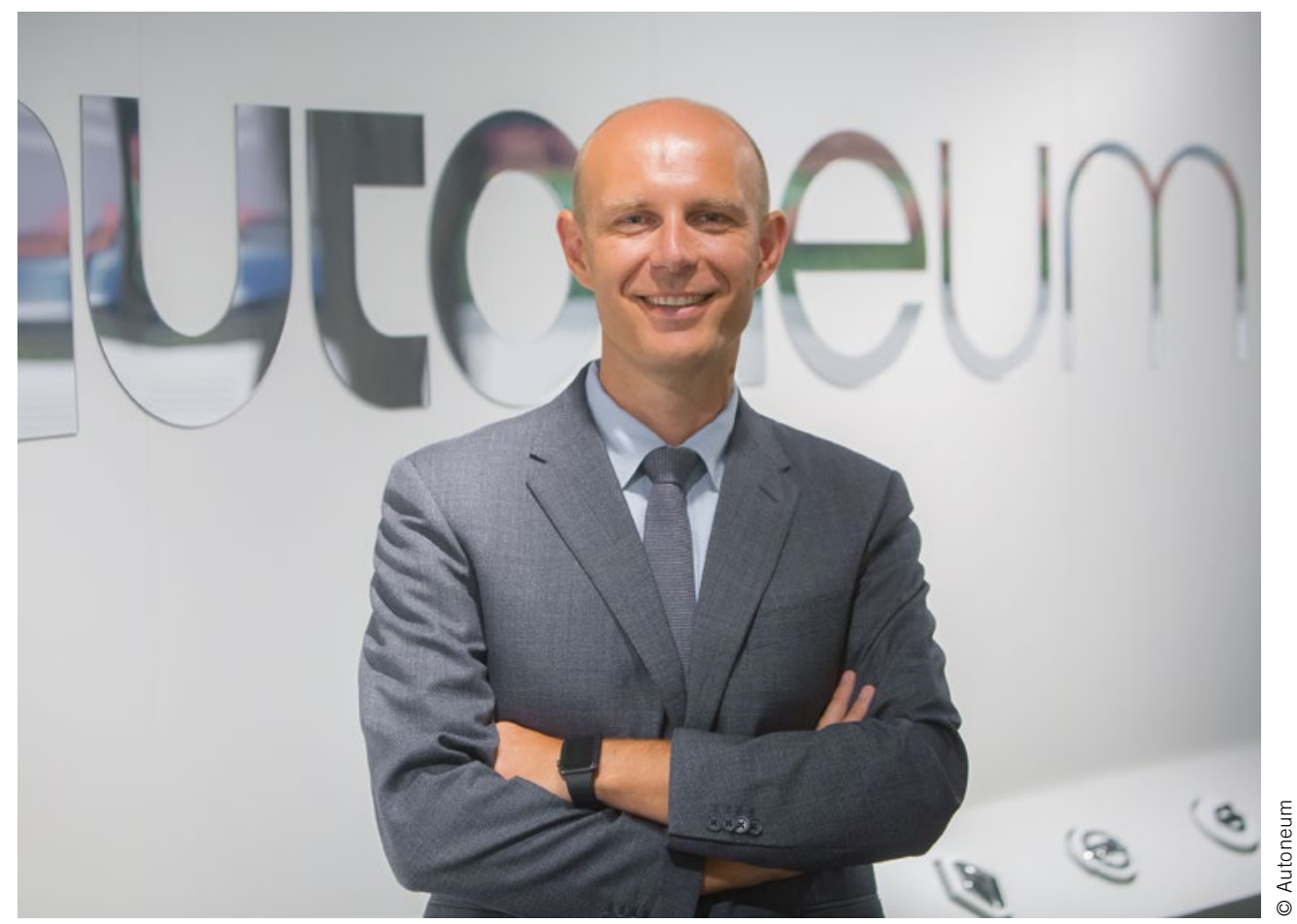

Dr. Davide Caprioli

Head of Product Acoustic and

Thermal Performance at Autoneum

\title{
The Evolution of Pass-by Noise Regulation
}

Road traffic noise is the most prevalent environmental noise source globally. With serious effects on the environment and on human health: Estimations show that in Europe alone, around $24 \%$ of the population is exposed to traffic noise in urban areas. This means that around 125 million people are likely to be exposed to threshold levels of road traffic noise according to the Environmental Noise Directive 2002/49/EC.

Throughout the last century, international standards and emission limits have been developed and adopted. In Europe, most of these initiatives focused on reducing traffic noise at the source by applying measures on new cars. Subsequently, limit values for Pass-by (PB) noise were lowered from $82 \mathrm{~dB}(\mathrm{~A})$ in 1970 to eventually $74 \mathrm{~dB}$ (A) in 1992. After a 15-year-long discussion to define a replacement for the ISO-362 measurement method, introduced in 1975, the UNECE working party on noise introduced regulation UN/ECE R51.03 in 2014. This regulation set $\mathrm{PB}$ noise limits to $72 \mathrm{~dB}$ (A) as of 2016 and also defined future emission reductions with limits of $70 \mathrm{~dB}$ by 2020 (phase 2) and $68 \mathrm{~dB}$ (A) by 2024 (phase 3).

Simultaneously, this regulation put an updated pass-by measurement method (ISO 362-1:2015) into effect: It calculates the final "pass-by noise level" as a weighted average of the noise emitted by the vehicle in a full-load acceleration and at constant speed, resulting in amore representative measurement of typical urban driving habits.
New methods and considerably reduced pass-by-noise limit values will challenge the automotive industry. PB noise homologation data that is currently available indicates that less than $30 \%$ of European cars complies with noise level limits set by phase 3 . However, $70 \%$ of all vehicles are in line with the phase 2 noise emission limit.

To close the gap between actual and allowed noise emissions, the automotive industry follows mainly two trends: Firstly, acoustic materials and components to encapsulate the sound source make powertrain noise practically negligible with regards to pass-by-noise. Here, the engineering challenge lies especially on efficient modular design and on finding the optimal ratio between material cost and weight of the acoustic package for different powertrain versions. Secondly, as far as tire noise is concerned, solutions for significant reductions (above $2 \mathrm{~dB}$ ) are not available yet, thus posing a real challenge for all the key players involved: OEMs, tire manufacturers and sound package suppliers.

The challenges mentioned above have to be solved by the automotive industry, especially given the fact that today certain European governments advocate stricter vehicle noise standards mostly from a cost-effective point-of-view: They are convinced that every Euro spent on the development and production of quieter vehicles is 100 times more effective in diminishing road noise than investing in improved road infrastructure. 